\title{
Le détournement de proverbes en FLE, transgression ou création ?
}

Syrine DIAZ-DAOUSSI, Université de Murcia, Espagne

\section{Introduction}

Les proverbes et autres unités phraséologiques demeurent actuellement assez peu enseignés en FLE mais une prise de conscience de l'importance de ces structures s'est faite progressivement à travers les indications du Cadre Européen Commun de Référence pour les Langues (CECR). Cependant, il est rare de trouver dans les manuels des activités qui proposent de «détourner» les proverbes. Nous proposerons donc des pistes de réflexion pratiques pour les professeurs de FLE qui désirent mettre en place des stratégies pour favoriser l'acquisition de ces structures et «transgresser » le proverbe originel pour permettre aux apprenants de développer leur créativité et de développer leur sensibilité humoristique dans la langue cible. Nous envisageons la langue en tant que langue-culture, concept didactique qui s'est forgé au fil des méthodologies d'enseignement /apprentissage fondées sur des conceptions différentes de la langue : par exemple, elle passe d'un ensemble de structures (méthode audiovisuelle) à un moyen de communication (approche communicative) pour enfin se transformer en un moyen d'interaction sociale au sens s'inscrivant dans une perspective décrite par Christian Puren comme co-actionnelle co-culturelle, synthétisé dans le schéma de Françoise Demougin. Chaque langue reflète un passé historique, social ou littéraire qui est à l'origine de la création d'une sphère linguistique, sociale et sémantique immédiatement compréhensible pour le locuteur natif qui a recours aux énoncés dits « idiomatiques », c'est-à-dire propres à une langue, de manière spontanée dans la mesure où «Chaque nation possédant un fondement socio-économique et historique se réfère à un réseau de significations et à un système de valeurs qui sont communs et semblent évidents aux locuteurs natifs. Tout écart par rapport aux expériences et valeurs connues risque fort d'être interprété de façon émotionnelle et négative, car ressenti comme comportement anormal » (Eisl 2).

Cette citation met en évidence la présence d'énoncés linguistiques culturellement marqués. C'est en quelque sorte la base de données commune à tous les locuteurs de la langue française dont le sens sera univoque car si nous utilisons l'expression «faire quelque chose pour des prunes », le locuteur non natif qui traduit littéralement cet énoncé se trouve en situation délicate d'incompréhension patente. Rares seront les locuteurs français qui 
connaîtront l'origine exacte de cette expression mais ils sauront l'utiliser de manière adéquate.

Dans le contexte scolaire, la culture étrangère est malheureusement délaissée au profit de l'enseignement de structures grammaticales par exemple, pour divers motifs comme le souligne Margit Eisl :

(...) le choix de la langue étrangère est de plus en plus motivé par les modes, la notion de l'utilité et la loi du marché. Dans ce contexte, la pratique quotidienne reste trop souvent très traditionnelle, l'enseignement de la langue étrangère se réduisant à l'acquisition de compétences grammaticales, fonctionnelles ou pragmatiques en communication, en fonction de l'enseignant(e) et des usages dans son entourage pédagogique. (3)

Les unités phraséologiques comme ici les proverbes peuvent donc s'avérer intéressantes comme vecteur culturel dans la mesure où elles permettent à l'apprenant de découvrir le réseau d'images mentales et sémantiques établi par les expressions idiomatiques dans la langue étrangère.

\section{Phraséologie, proverbes, proverbes détournés : présentation}

Nous commencerons dans un premier temps par aborder le concept d'unité phraséologique dans la mesure où il s'agit d'un concept clé pour pouvoir parler par la suite de transgression linguistique.

Dès la fin du dix-neuvième siècle, les linguistes allemands, anglais et français ont commencé à s'intéresser aux constructions stables «caractérisées par leur structure d'éléments réagroupés formant un bloc, employées avec réitération et signifiant tout autre chose que ce que chaque constituant signifie séparément »(Gonzalez-Rey 21).

C'est d'abord en Allemagne, avec Paul que s'ébauche ce concept, repris ensuite sous le nom d'idioms en anglais. Pour le français, Bréal met en évidence l'importance de «formules », «locutions », « groupes articulés », caractérisés par le figement et l'opacité sémantique de leurs composants (23).

Nous remarquons donc qu'une unité phraséologique est un concept vaste qui se réfère d'une part à la morphosyntaxe d'une suite de mots (polylexicalité) et d'autre part à leur caractère idiomatique, c'est-à-dire propre à chaque langue. Nous déduisons donc qu'il s'agit d'une suite de mots stables et figés qui ne tolèrent pas la transgression a priori si nous voulons être compris par un locuteur natif.

Les proverbes, et de manière générale tous les énoncés connexes englobés sous l'archilexème «parémies » comme le souligne Julia Sevilla Muñoz sont considérés comme le 
reflet de la sagesse populaire, comme l'atteste le dictionnaire Larousse (2010) puisque le proverbe est défini comme étant un énoncé exprimant un «conseil populaire ». Sémantiquement parlant, il exprime donc une vérité générale, admise par tous et au niveau structurel, Maria Isabel Gonzalez Rey souligne son appartenance au groupe des énoncés phraséologiques : «(..) les parémies sont pourvues d'une structure syntaxique, d'une valeur sémantique et d'une fonction pragmatique qui les caractérisent comme expressions figées, (...) au même titre que les collocations et les expressions idiomatiques ».

Gonzalez Rey met ainsi en évidence la présence de certains traits définitoires des proverbes à un triple niveau (syntaxe, sens et pragmatique) et souligne également «(...) leur caractère d'unités de sens à structure binaire, éléments mnémotechniques et valeurs sentencieuse, entre autres »(74).

Elle met ainsi en évidence le premier critère de définition d'ordre sémanticosyntaxique lorsqu'elle évoque des «unités de sens à structure binaire ». C'est ce que l'on dénomme par le concept de «figement» sur lequel nous reviendrons plus tard. L'aspect phonétique est également important puisque c'est la rime qui aidera le locuteur à se souvenir du proverbe comme dans le cas de « La nuit, tous les chats sont gris ».

\subsection{Les proverbes : des unités phraséologiques complexes et difficiles à classer (Salah Mejri).}

Les proverbes reposent, comme nous l'avons vu précédemment, sur une structure binaire qui peut jouer sur une rime, établissant ainsi un parallélisme entre le premier segment et le second. Mejri met en évidence les caractères prosodiques des proverbes dans la mesure où « (...) le proverbe se présente comme un énoncé obéissant à une fixité rythmique qu'on peut ramener à une structure le plus souvent binaire combinant assonances et allitérations appuyées par un « retour périodique d'éléments marqués » (Rodegem 122) pouvant avoir la configuration d'une rime interne.

Cette citation met en évidence la double nécessité de travailler avec les apprenants sur un plan à la fois sémantique (signification des termes) aussi bien que sur un plan prosodique (rythme, forme, rimes) dans la mesure où l'alliance de ces deux éléments permet de saisir le proverbe dans toute son idiomaticité : cette étape est primordiale afin d'amener les apprenants à se familiariser avec un proverbe étranger, de le comprendre, de l'identifier et par la suite, de pouvoir se l'approprier et le détourner.

Il est important de remarquer que les parémies n'ont pas le même statut en français qu'en espagnol : en effet, elles sont beaucoup plus fréquentes en espagnol qu'en français, comme le souligne la linguiste Julia Sevilla Muñoz qui s'est longuement consacrée à leur 
étude de manière contrastive : «On sait par exemple, qu'il y a une différence dans leur usage dans les langues comme le français et l'espagnol, car les proverbes sont plus utilisés dans ce dernier que dans la langue de Jean de La Fontaine ».

Cette citation met en exergue un usage moindre des parémies en français au niveau quantitatif qu'en espagnol par exemple, ici, la langue maternelle des apprenants comme le souligne Sevilla Muñoz mais leur maîtrise semble relativement importante pour des apprenants de FLE dans la mesure où ils comblent un besoin culturel et communicatif en partageant le patrimoine linguistique commun des locuteurs français.

\subsection{Le proverbe, aboutissement de l'unité lexicale}

\begin{tabular}{|c|c|c|}
\hline unité lexicale simple $\rightarrow$ & unité dérivée $\rightarrow$ unité syntagmatique $\rightarrow$ & Proverbe, \\
\hline roi & être plus royaliste que le roi & $\begin{array}{l}\text { Il ne faut pas être } \\
\text { plus royaliste que le } \\
\text { roi }\end{array}$ \\
\hline
\end{tabular}

Ce schéma reflète le statut particulier du proverbe comme l'évolution la plus aboutie d'une unité lexicale isolée par le biais de la dérivation à partir d'un substantif. Le proverbe acquiert donc toute son autonomie discursive ou peut être inséré dans le discours mais ne peut pas être généralisé pour toutes les structures.

\subsection{La notion de figement}

Lorsque nous parlons d'expression «figée », cet adjectif est synonyme de solidifié. Une unité phraséologique est dite figée lorsqu'elle est utilisée par une grande majorité de natifs et qu'elle fait partie de la norme, comme c'est le cas des proverbes. Gaston Gross s'est intéressé à la notion de figement qu'il définit à partir de plusieurs critères, à savoir :

1. la polylexicalité :

La première condition nécessaire pour qu'on puisse parler de figement est que l'on soit en présence d'une séquence de plusieurs mots et que ces mots aient, par ailleurs, une existence autonome (9)

Il est donc important de faire prendre conscience à nos apprenants qu'une séquence dite figée se construit sur la base de plusieurs lexies concomitantes qui possèdent un sens différent lorsque nous les cherchons dans le dictionnaire, par exemple.

2. l'opacité sémantique :

Prenons l'exemple donné par Gross concernant une expression imagée, c'est-à-dire une expression idiomatique qui a recours à une métaphore pour exprimer une notion ou un 
concept «la moutarde lui monte au nez. »Cette suite de mots peut paraître obscure voire opaque pour une personne qui apprend le français.

Le sens ordinaire des mots de cette phrase ne permet pas de conclure que la phrase dans son ensemble signifie que l'on parle d'une personne qui se fâche. (11)

En effet, le locuteur non-natif (hispanophone ici) connaît certainement tous les éléments de la phrase de manière isolée : moutarde (mostaza)- monter (subir)- nez (nariz) mais ces trois éléments forment ici un bloc sémantique indivisible qui peut constituer un obstacle à la compréhension. En effet, la phrase ne peut pas être interprétée de manière littérale et dans ce cas, la phrase est considérée comme n'ayant pas de lecture compositionnelle. Une phrase qui a une lecture compositionnelle serait : Le chien aboie dans le jardin. Ici, la phrase est claire et n'admet qu'une seule interprétation. Dans le cas des proverbes, un énoncé comme «Qui ne dit mot consent» est plus transparent au niveau sémantique que «A vieille mule, frein doré » pour un apprenant dans la mesure où la métaphore suppose un niveau de compréhension plus complexe à déchiffrer.

3. le blocage des propriétés transformationnelles :

Les phrases compositionnelles acceptent plusieurs transformations d'ordre grammatical comme le passage à la voix passive, la pronominalisation, ou encore l'insertion d'un pronom relatif. En revanche, cette règle n'est pas valable pour les phrases noncompositionnelles, comme le souligne Gross : «Or, ce qu'il faut souligner avec insistance, c'est que l'opacité sémantique et les restrictions syntaxiques vont de pair ». (12). Lorsqu'il transforme la suite «Luc a pris la tangente » à la voix passive, elle est incorrecte : *La tangente a été prise par Luc.

Nous pouvons donc remarquer, à la lecture de cette citation, que les suites noncompositionnelles sont moins souples que les autres et leurs propriétés transformationnelles sont bloquées.

\subsection{Le proverbe détourné. Définitions}

Que l'on parle de proverbe « détourné », « dérivé » ou encore «tordu » en français, en espagnol «refrán alterado » ou l'anglais « anti-proverb » pour reprendre la terminologie utilisée par Péter Barta, il apparaît clairement que le proverbe détourné évoque la transgression de l'unité phraséologique initiale, voire qu'il va carrément à son encontre comme c'est le cas de l'anglais.

Barta définit le proverbe détourné comme une «parodie du proverbe derrière lequel se cache soit un proverbe concret $(\ldots)$, soit un schéma proverbial $(\ldots) »(140)$. 
Les critères de la transgression linguistique sont alors soit d'ordre sémantique, soit d'ordre formel. En effet, une partie est défigée, c'est-à-dire que le locuteur transforme une partie de l'unité phraséologique selon son contexte, sa réalité, alors que l'autre conserve son figement lexical ou rythmique afin de permettre aux locuteurs qui l'écoutent ou le lisent d'identifier le proverbe originel.

Audrey Wozniak souligne que cette transgression est aussi personnelle qu'infinie puisque «(..) un seul être, (...) en un instant et un lieu donnés choisit d'intervenir formellement sur un matériau relativement fixe de sa compétence linguistique ».

Les procédés de détournement de proverbes sont nombreux et Barta en cite quelques exemples, en commençant par la paronomase :

La vérité sort de la couche des enfants < La vérité sort de la bouche des enfants.

Dans cet énoncé, le procédé de détournement consiste à remplacer un terme par un autre qui en est très proche au niveau des sonorités / u $\int_{0} /$ en changeant le premier phonème. L'aspect comique du proverbe détourné repose sur le jeu de mots ainsi que sa plausibilité (un enfant est susceptible de remplir sa couche...de quelque chose de concret pour ainsi dire.)

Le second procédé de détournement est le recours à la polysémie : Barta nous donne l'exemple du proverbe détourné suivant :

Deux sûretés valent mieux qu'une $<$ Deux précautions valent mieux qu'une.

Le terme précaution a été remplacé par un de ses synonymes qui défige complètement le proverbe, créant ainsi un jeu de mots prêtant à sourire.

Le troisième procédé cité par Barta est l'interprétation de l'abstrait (du figuré) par le

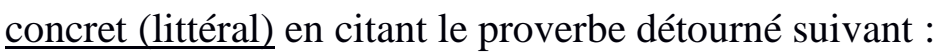

Tant va la cruche à l'eau qu'à la fin....elle s'emplit < Tant va la cruche à l'eau qu'à la fin elle se casse.

Dans ce cas de figure, la seconde partie du proverbe est remplacée par une autre qui devient automatiquement défigée et compositionnelle, perdant ainsi son caractère proverbial et s'opposant sémantiquement à l'énoncé originel.

Le quatrième procédé de détournement est l'homophonie, comme l'illustre cet exemple :

Dis-moi qui tu hantes et je te dirai qui tu hais < dis-moi qui tu hantes, je te dirai qui tu es.

Ce jeu de mots est uniquement visible à l'écrit car l'homophonie ne permet pas de déterminer à l'oreille si le proverbe a été détourné. Il crée l'humour de ce fait et serait susceptible d'apparaître dans un article de presse, par exemple. 
Le dernier procédé cité par Barta est l'adjonction, comme c'est le cas de

L'argent ne fait pas le bonheur...mais il y contribue »<L'argent ne fait pas le bonheur.

En effet, un syntagme s'ajoute au proverbe et lui donne un aspect matériel et très pragmatique qui rompt avec les idéaux traditionnellement rattachés à ce proverbe (d'ordinaire, l'aspect romantique l'emporte sur l'aspect matériel). Toutes ces structures idiomatiques font partie intégrante de la langue étrangère et occupent une place privilégiée au sein de la didactique des langues au niveau du Conseil de l'Europe qui encourage l'enseignement/apprentissage de ces structures, comme nous allons le voir à présent.

\section{Un enseignement phraséologique encouragé par le CECR}

Le CECR est un document européen rédigé en 2001 et dont le but principal est d'une part d'uniformiser toutes les équivalences relatives aux niveaux de langue en Europe, notamment par le biais de descripteurs correspondant à des compétences (expression orale, compréhension orale, expression écrite, production écrite) sur une échelle allant de A1 (débutant) à C2 (considéré comme très proche d'un locuteur natif). D'autre part, le CECR souhaite insister sur l'importance de l'aspect communicatif lors de l'enseignement/apprentissage des langues étrangères, en amenant les apprenants à être capables d'autonomie dans des situations de la vie courante. Ceci passe notamment par la maîtrise des structures idiomatiques de chaque langue, considérées comme déterminantes dans l'apprentissage selon plusieurs linguistes (Gonzalez Rey et Sevilla Muñoz par exemple). En effet, la maîtrise des énoncés phraséologiques démontre une certaine habileté linguistique qui rapproche de locuteur non-natif du natif, puisqu'il adopte des attitudes courantes du second. De ce fait, les proverbes sont considérés comme des expressions de la sagesse populaire qui « renforcent des attitudes courantes » par le CECR, qui nous donne l'exemple suivant : Un «tiens » vaut mieux que deux « Tu l'auras »! (87).

Ce que le CECR qualifie d' «attitudes courantes » sont bien entendu les attitudes utilisées par les natifs lorsqu'ils utilisent leur propre langue, ici les natifs français qui vont spontanément avoir recours à ces structures idiomatiques afin de commenter, de ponctuer ou d'illustrer une situation donnée. L'utilisation des structures figées de la langue témoigne de deux compétences langagières, à savoir dans un premier temps la compétence linguistique (lexicale et grammaticale) ainsi que la compétence socioculturelle.

Au niveau des outils que nous pourrions proposer aux apprenants, il existe un certain nombre de sites Internet pour travailler en classe, en autonomie ou simplement pour 
découvrir de nouveaux proverbes, comme le site l'Internaute qui propose un dictionnaire de proverbes regroupés par thèmes ${ }^{1}$.

Il existe également un site internet du Centre Européen pour les Langues vivantes qui propose de faire découvrir de manière collaborative des proverbes regroupés par langues et d'en proposer une explication avec des mots simples, accessibles sur le site [http://archive.ecml.at/interactive/sentences.asp].

Enfin nous proposerons à nos apprenants de consulter le « Refranero multilingue » de l'Institut Cervantès. Il s'agit d'une base de données parémiologiques multilingue où l'apprenant peut rechercher un proverbe par l'idée clef qu'il contient et accéder à une fiche qui retrace sa récurrence littéraire ou encore sa fréquence d'utilisation. Ce site est accessible sur [http://cvc.cervantes.es/lengua/refranero/Busqueda.aspx].

Toutefois, ces structures figées peuvent être amenées à être transgressées par le biais du défigement, qui constitue «une manipulation lexicale, syntaxique ou sémantique de ces unités. Ces différences formelles entraînent une modification du sens » (Català 62).

Nous considérons le défigement comme une forme de transgression linguistique dans la mesure où il s'éloigne de la norme linguistique incarnée par un énoncé phraséologique stable et figé dans une langue donnée. Si l'on souhaite travailler en classe sur le détournement de proverbes, il est important d'avoir recours à des documents authentiques, que ce soit des textes littéraires (nous pensons notamment à l'OULIPO et au surréalisme), ou encore des textes journalistiques et publicitaires qui mettront en avant des énoncés détournés dans le but d'attirer l'attention du lecteur par le biais d'un jeu de mots.

Détourner des proverbes est une activité de création de la part de l'apprenant qui possède déjà une certaine maîtrise de la langue cible. En ce sens, Cristelle Cavalla considère que l'apprentissage des unités phraséologiques peut se faire à partir d'un niveau intermédiaire, c'est-à-dire à partir du niveau B sur l'échelle établie par le CECR : «La phraséologie apparaît à partir du niveau $\mathrm{B}$ et doit être maîtrisée à la fin des niveaux C. » (Cavalla).

L'intérêt de détourner les proverbes réside dans la créativité dont peut faire preuve l'apprenant en établissant des jeux de mots tout en lui permettant de «Surprendre l'interlocuteur et créer une atmosphère de connivence (...) par le biais d'une connaissance partagée (...) » (Català 62).

En effet, cette «connaissance partagée » renvoie au fonds linguistique et culturel commun propre à chaque langue mentionnée par Eisl. En maîtrisant les énoncés 
phraséologiques ainsi que les codes de leur transgression, le locuteur non natif est très proche de la sphère culturelle du natif.

\section{Application didactique: cas pratique}

En classe, nous pourrions envisager l'enseignement/apprentissage de la phraséologie pour un public adulte hispanophone (universitaire) qui posséderait un niveau compris entre $\mathrm{B} 1$ et $\mathrm{B} 2$.

L'enseignement de proverbes détournés ou du moins de stratégies pour détourner un énoncé proverbial phraséologique possède un intérêt certain, dans la mesure où les apprenants sont amenés à transgresser d'une part des énoncés figés et d'autre part, à développer leur créativité, en respectant toutefois des conditions d'ordre syntaxique ou rythmique qui permettront d'identifier le proverbe initial. En classe, nous nous sommes demandé comment familiariser les apprenants avec ce type de structure par le biais de documents authentiques et de manière fluide. L'approche onomasiologique est relativement récente (nous citons l'exemple de Cavalla et Crozier) dans la mesure où elle envisage l'enseignement du lexique en le regroupant par notions. Cette approche donne donc à l'apprenant des outils pour exprimer tel sentiment ou telle notion, indépendamment de la structure formelle des unités présentées. En effet, l'approche onomasiologique «permet d'aborder non pas les expressions contenant, par exemple, le nom triste mais celles signifiant triste ou de façon plus générale, comment dire la tristesse. Par exemple, la collocation avoir le cafard ne contient pas le mot triste mais participe à l'expression de la tristesse » (Cavalla 7).

Nous retenons donc que ce sont les réseaux sémantiques qui existent entre les mots et non pas tous les dérivés d'une même famille qui permettent aux apprenants d'enrichir l'étendue de leur lexique en le regroupant par notions.

Cette approche permet d'aborder un lexique pour évoquer un concept défini et mène l'apprenant à l'autonomie langagière dans la mesure où «L'entrée par concept tend à mener l'apprenant vers « [u]ne autonomie fonctionnelle en L2 [qui] suppose le développement de liens rapides et efficaces entre le niveau lexical de L2 et les concepts sous-jacents, ce qui évite à un apprenant de passer par le lien de la traduction en L1. » (Frenck-Mestre et al 61). En d'autres termes, c'est en comprenant les concepts sous-jacents aux lexies que les apprenants traduiront moins systématiquement, car ils se rendront compte que la traduction n'est soit pas possible soit pas satisfaisante pour plusieurs raisons (syntaxique, lexicale, sémantique et culturelle) (Cavalla 8). 
Cette approche nous semble intéressante car elle fait en sorte de ne pas recourir à la traduction systématique en langue maternelle et développe l'autonomie de l'apprenant.

Notre proposition s'adresse à un public adulte hispanophone de niveau B2 et s'articule au sein d'une séquence intitulée «parler de soi, de ses habitudes » où les apprenants seront amenés à travailler par binômes ou de manière individuelle, à partir de documents authentiques (des articles de presse notamment). Les apprenants ont déjà été confrontés au proverbe originel en réalisant dans un premier temps une étape de repérage guidé des proverbes en vérifiant s'ils ont bien compris leur signification (est-il compositionnel ou non ?) par le biais de l'expression orale en interaction, où chaque élève peut donner son opinion et nuancer les propos de l'autre.

Prenons l'exemple d'un article publié le 27 avril 2009 dans le Figaro.fr intitulé «L'avenir appartient à ceux qui se lèvent tard $»^{2}$ qui possède la particularité de présenter sur un même support journalistique le proverbe détourné et le proverbe originel (le monde appartient à ceux qui se lèvent tôt). L'article en question évoque les différences de concentration au long d'une journée entre les personnes qui se lèvent tôt et les autres qui se lèvent plus tard. Ces dernières seraient plus auraient moins de mal à fixer leur concentration durant la journée.

\section{Étapes de la séance de cours}

Dans un premier temps, nous proposerions une étape de compréhension globale (lecture individuelle et mise en commun à l'oral).

Ensuite, nous demanderions aux élèves de repérer les unités phraséologiques originelles et détournées. Il conviendra de préciser que le proverbe originel est flexible sur le plan lexical car il admet des variantes (le monde/l'avenir).

La phase d'expression orale servira à la reformulation des proverbes (pré-requis) afin de nous assurer que tous les élèves aient bien compris leur sens en les utilisant en contexte.

Nous travaillerons ensuite sur le procédé de détournement utilisé en demandant aux élèves de quelle manière le proverbe originel a été transformé (a-t-on joué sur les sons, ajouté ou remplacé une partie du proverbe ?). Précisons que nous préférons éviter tout technicisme en classe et aurons recours à une terminologie simplifiée et non spécialisée. Nous souhaitons en effet que les élèves soient conscients des phénomènes qu'ils pourront exprimer avec leurs propres mots plutôt que d'entrer dans une multiplicité de termes.

Par la suite, nous leur proposerons un repérage non-guidé de proverbes détournés issus de «l'avenir appartient à ceux qui se lèvent tôt » repris dans d'autres articles (par exemple, «En rugby, l'avenir appartient à ceux qui courent très vite »). 
Enfin, nous les amènerons à manipuler les proverbes par binômes, en nous inspirant du jeu des «proverbes mutants » cité par Françoise Vanhemelryck où le jeu consiste à «changer un ou plusieurs éléments (mots) dans des proverbes existants et de créer ainsi un nouveau proverbe. Les éléments à changer seront marqués ou non ».

Les compétences de l'apprenant qui sont développées sont principalement linguistiques (lexicales et grammaticales), les savoirs sont appréhendés de manière ludique.

En définitive, les proverbes détournés, même s'ils représentent une transgression des unités phraséologiques françaises, au sens où ils s'éloignent de l'énoncé figé dans la langue source qui en fait un énoncé normé, permettent à l'apprenant de manipuler la langue et de développer sa créativité. Il est très important de travailler à la fois en contexte afin d'associer un proverbe à une situation donnée mais également de privilégier une approche onomasiologique afin d'aborder les savoirs de manière transversale par le biais de notions qui permettront à l'apprenant d'acquérir une autonomie langagière en rattachant un réseau de structures figées à une même notion.

Il est également crucial de bien s'assurer que les élèves maîtrisent correctement le proverbe initial avant de proposer de le détourner. Détourner, transgresser ou s'éloigner de la norme doit se faire en respectant certains aspects du figement (conserver une rime ou un parallélisme structurel par exemple) afin que l'interlocuteur puisse reconnaître le proverbe initial.

\section{Bibliographie}

Anscombre, Jean-Claude. Les proverbes: un figement du deuxième type ? (2005). http://linx.revues.org/255

Barta, Péter. «Au pays des proverbes, les détournements sont rois. Contribution à l'étude des proverbes détournés du français (II)». Paremia (2006). http://www.paremia.org/paremia/06-Barta.pdf

Català Guitart, Dolors. «Figement et défigement des proverbes comme outil didactique du FLE ». Paremia (2006). www.paremia.org/wp-content/uploads/06-CATALA.pdf

Cavalla, Cristelle. «La phraséologie en classe de FLE ». Les langues modernes 1 (2009). http://www.aplvlanguesmodernes.org/IMG/pdf/2009-1_cavalla.pdf

Conseil de l'Europe. Cadre européen commun de référence pour les langues. 2001. http://www.coe.int/t/dg4/linguistic/CADRE_FR.asp

Demougin, Françoise. «La didactique des langues - cultures à la croisée des méthodes ». Tréma 30 (2008). http://trema.revues.org/427 (consulté le 22/12/2014). 
Eisl, Margit. «La perspective interculturelle en FLE : des principes didactologiques aux activités de classe (enseignement secondaire autrichien) ». Travaux de didactique $d u$ français langue étrangère 54 (2006).

González-Rey, Maria Isabel. La phraséologie du français. Toulouse: PU du Mirail, 2002.

Gross, Gaston. Les expressions figées en français. Paris : Ophrys, 1996.

Mejri, Salah. «Inférence et structuration des énoncés proverbiaux ». 2001. www.halshs.archives-ouvertes.fr/docs/00/41/13/.../S.MEJRI.Les_proverbes.pdf

Sevilla Muñoz, Julia. «Les proverbes et phrases proverbiales français, et leurs équivalences en espagnol ». Langages 139 (2000) : 98-109.

Vanhemelryck, Françoise. «Comment exploiter des documents publicitaires en classe de FLE ? ». Romaneske 2 (1994) : 28-52.

Wozniak, Audrey. «Le proverbe détourné : étude théorique appliquée à un corpus bilingue franco-espagnol ». Paremia (2009) : 185-196.

\section{NOTES}

${ }^{1}$ [http://www.linternaute.com/proverbe/theme/]

2 [http://www.lefigaro.fr/sciences/2009/04/27/01008-20090427ARTFIG00394-1-avenir-appartient-a-ceux-quise-levent-tard-.php] 